Int. J. Morphol.,

31(1):184-188, 2013.

\title{
Estudio Morfométrico del Nervio Óptico de Tiburoncito (Ariopsis seemanni)
}

\author{
Morphological Study of Tete Sea Catfish (Ariopsis seemanni) Optic Nerve
}

Diana Carolina Castañeda Cortés; Oswaldo Tovar Bohórquez \& Hernán Hurtado Giraldo

\begin{abstract}
CASTAÑEda, C. D. C.; TOVAR, B. O \& HURTADO, G. H. Estudio morfométrico del nervio óptico de tiburoncito (Ariopsis seemanni). Int. J. Morphol., 31(1):184-188, 2013.

RESUMEN: En peces, el nervio óptico es el encargado de transportar la información integrada por las células ganglionares de la retina hacia el tectum óptico, para que se generen imágenes acerca del entorno. El objetivo de este trabajo es describir morfométricamente el nervio óptico del tiburoncito (Ariopsis seemanni), para lo cual se utilizó la Microscopía óptica de Alta Resolución (MOAR), realizando cortes a 1 micra de espesor. El nervio óptico de A. seemanni presenta fibras mielínicas de diverso calibre, acompañadas de oligodendrocitos y astrocitos. El nervio está cubierto por las meninges, que presentan vasos sanguíneos y adipocitos. El nervio tiene un área total de $179604 \pm 30163 \mu \mathrm{m} 2$, diámetro de $478 \pm 42 \mu \mathrm{m}$ y un número total de fibras mielínicas de $22848 \pm 4350$, de las cuales la mayoría tiene un tamaño pequeño, que puede estar relacionado con una velocidad de conducción baja.
\end{abstract}

PALABRAS CLAVE: Tiburoncito; Ariopsis seemanni; Nervio óptico; Fibras nerviosas; Morfometria.

\section{INTRODUCCIÓN}

El sistema visual de las especies que viven en ecosistemas complejos tiene características que le permiten adquirir, enfocar, trasmitir y crear la imagen más precisa del objeto percibido, por medio de diferentes adaptaciones estructurales que pueden diferir entre las especies (Collin \& Shand, 2003; Fernald, 2000). La energía lumínica que viene proyectada desde el cristalino es captada por los fotoreceptores, que la convierten en energía electroquímica, la cual se transfiere a una serie de interneuronas que la trasladan finalmente a las células ganglionares, cuyos axones conforman el nervio óptico y trasportan las señales al cerebro para ser interpretadas (Collin \& Shand, 2003; Sanes \& Zipursky, 2010). Tanto para peces, como para otros vertebrados, se ha descrito que en el nervio óptico existen subpoblaciones de fibras mielínicas que difieren en su tamaño y velocidad de conducción (Akaishi et al., 1995; Donovan, 1967; Easter et al., 1981; Guthrie \& Banks, 1990; Sanchez et al., 1986; Woodbury \& Ulinski, 1986).

Ariopsis seemanni (Günther, 1864) es un pez que habita en aguas dulces costeras, aguas salobres y saladas del Océano Pacífico. Hace migraciones temporales de ambientes salobres hacia estuarios y aguas dulces (Bussing, 1998). Habita ambientes muy complejos y variados como mangla- res y estuarios (Robertson \& Allen, 2002), en los cuales la entrada de luz no es uniforme, teniendo variaciones en la longitud de onda, intensidad y dispersión. De acuerdo a lo anterior, el ojo de A. seemanni debe presentar adaptaciones que le permitan interactuar con dicho ambiente. Uno de los aspectos importantes al respecto, es el número de fibras mielínicas que se encuentran en el nervio óptico, ya que a través de estas fluye información visual hacia los centros de integración e interpretación en el cerebro. Teniendo en cuenta esto, en este trabajo se definen los rangos de tamaños de fibras mielínicas en el nervio óptico de $A$. seemanni, para contribuir al conocimiento del sistema visual de esta especie.

\section{MATERIAL Y MÉTODO}

Se utilizaron 7 ejemplares juveniles de A. seemanni con un peso promedio de $21.57+/-5.02 \mathrm{~g}$ y una longitud estándar promedio de $10.63+/-0.98 \mathrm{~cm}$. Los ejemplares fueron comprados a un distribuidor de peces ornamentales y mantenidos en el Laboratorio de Acuicultura de la Universidad Militar Nueva Granada, en sistemas de recirculación, alimentados ad libitum con truchina al $45 \%$ 
de proteína tres veces por día. Los peces fueron anestesiados con benzocaina $(0.1 \mathrm{~g} / \mathrm{L})$ y sacrificados por corte medular a nivel cervical (CCAC. Canadian Council on Animal Care, 2005). Se realizó la disección, tomando muestras de nervio óptico de la región cercana a la esclera. Las muestras se procesaron siguiendo metodologías ya publicadas (Caldas et al., 1993). Brevemente, las muestras se fijaron en mezcla de formaldehido y glutaraldehido, se posfijaron con tetraóxido de Osmio, se deshidrataron en concentraciones ascendentes de etanol, se impregnaron y polimerizaron en resina Poli/Bed $812 ®$. Finalmente se realizaron cortes trasversales a $1 \mu \mathrm{m}$ de espesor, y se tiñeron con azul de toluidina. Para el análisis morfométrico, se procedió a la captura de imágenes, en las cuales se midió el área del nervio, se contó el número de fibras mielínicas, y se obtuvo el área y díámetro de esas fibras con el software ScionImage (http://www.scioncorp.com/): para cada individuo, aleatoriamente se seleccionaron 5 fotografías de diferentes regiones del nervio, en un aumento de 800x. Estas fotografías fueron impresas y se indicaron las fibras mielínicas con un punto. Estas fueron encerradas en un círculo que incluía la mielina. Se escanearon las impresiones y con el software se contaron las fibras, se midió el área muestreada, y se calculó el número de fibras para el área total del nervio. De las fibras seleccionadas se obtuvo el área y el diámetro. Se realizaron histogramas de frecuencia de tamaño, definiendo los rangos de tamaño para áreas cada cinco micras y para los diámetros cada micra.

\section{RESULTADOS}

El nervio óptico de A. seemanni está cubierto por las meninges, en las cuales se observa la arteria central, vasos sanguíneos y adipocitos. Se presentan septos de tejido conectivo vascularizado entrando al tejido del nervio. También se encontraron septos gliales más pequeños que los septos de tejido conectivo. En estos septos gliales se identificaron astrocitos y oligodendrocitos. El nervio óptico de $A$. seemanni presentó un área total de $179600 \pm 30163 \mu \mathrm{m}^{2}$, diámetro de $478 \pm 41 \mu \mathrm{m}$ y un número total de fibras mielínicas de $22848 \pm 4350$. Estas fibras tenían una gran variedad de tamaños que iban desde los $0,5 \mu \mathrm{m}^{2}$ hasta 186 $\mu \mathrm{m}^{2}$ de área (Tabla I). El rango que presentó el mayor nú- mero promedio de fibras fue el de 0,5 a $12 \mu \mathrm{m}^{2}$, con $4455 \pm 527$ y el que presentó el menor número fue el rango de $<34 \mu \mathrm{m}^{2}$, con $83 \pm 57$ fibras.

En cuanto a diámetro de las fibras nerviosas el rango de 0,5 a $4 \mu \mathrm{m}$ presentó el mayor número de fibras (4552 \pm 532 ). El menor número de fibras fue de $47 \pm 14$ (rango de $<8 \mu \mathrm{m}$ ) (Tabla I).

\section{DISCUSIÓN}

El nervio óptico de A. seemanni presenta fibras mielínicas empaquetadas en fascículos. Estas fibras son las prolongaciones axónicas de las células ganglionares de la retina y están encargadas de llevar la información que captan las células ganglionares de la retina hacia el cerebro, para que este la interprete y pueda generar una respuesta (Butler \& Hodos, 2005; Collin \& Shand, 2003; Fernald, 2000; Wullimann et al., 1996). Al igual que en la mayoría de los vertebrados, el nervio está recubierto por tres capas de tejido conectivo: la piamadre, aracnoides y la dura madre, que corresponden a las capas meníngeas respectivas que recubren el cerebro (Fig, 1) (Fujita et al., 2000; Maggs \& Scholes, 1990; Easter et al., 1981).

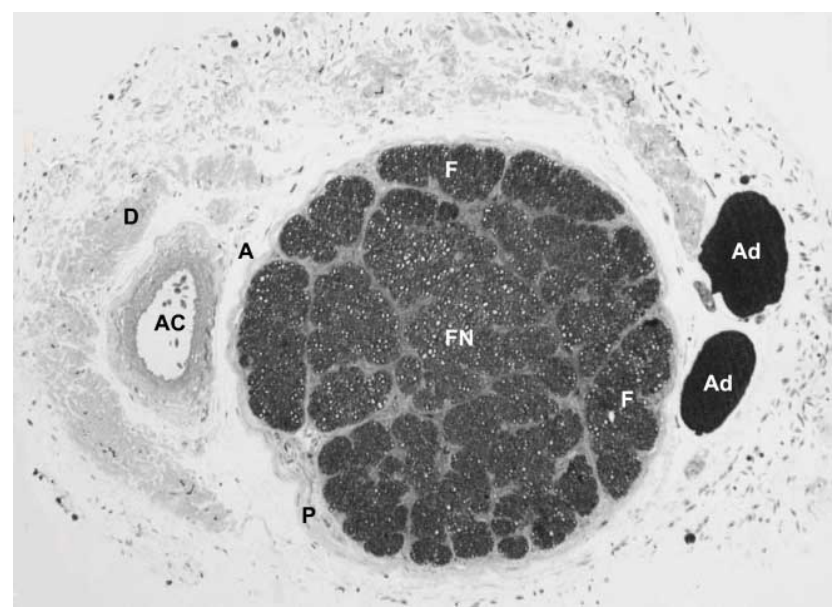

Fig. 1. Corte trasversal del nervio óptico de A. seemanni. Fibras Nerviosas (FN); Fascículos (F); Pia madre (P); Aracnoides (A); Dura madre (D); Arteria central (AC); Adipositos (Ad). Azul de Toluidina. 80X. UMNG. 2009.

Tabla I. Número de fibras mielínicas presentes para los diferentes rangos de área y diámetro del nervio óptico de A. seemanni.

\begin{tabular}{lccc}
\hline Rangos Area $\left(\mu \mathbf{m}^{2}\right)$ & $\mathbf{0 . 5 - 1 2}$ & $\mathbf{1 2 . 5 - 2 4}$ & $<\mathbf{2 4}$ \\
Número fibras $($ media \pm D.S) & $4455 \pm 527$ & $321 \pm 195$ & $83 \pm 57$ \\
Rangos diámetro $(\mu \mathbf{m})$ & $\mathbf{0 . 5 - 4}$ & $\mathbf{4 . 5 - 8}$ & $<\mathbf{8}$ \\
Número fibras (media \pm D.S.) & $4552 \pm 532$ & $260 \pm 103$ & $47 \pm 14$ \\
\hline
\end{tabular}


La disposición que presentan los septos gliales (en medio de las fibras nerviosas), según lo reporta la literatura, ayuda a que los elementos nerviosos permanezcan aislados del tejido conectivo y de los vasos sanguíneos en todo el nervio óptico (Butt et al., 1994; Maggs \& Scholes, 1990). En estos septos, se diferenciaron dos de los principales tipos de células gliales, los oligodendrocitos y los astrocitos. Los primeros se encargan de construir las vainas mielínicas de los axones. Los segundos tienen varias funciones, una está relacionada con la formación de gliales limitantes perivasculares y subpiales, creando agrupaciones de fibras nerviosas. También participan en procesos perinodales, permitiendo contactos estrechos con la membrana del axón en los nodos de Ranvier (Bolton \& Butt, 2005; Butt et al., 2004; Maggs \& Scholes, 1990) lo cual es importante en la regulación de las concentraciones de potasio $\mathrm{K}+$, implicadas en el potencial de acción (Bolton \& Butt, 2005; Hoppe et al., 1991).

En peces, el tamaño del nervio óptico y el número de fibras mielínicas presentes en él es muy variable. En $A$. seemanni presenta un diámetro promedio de 478,2 \pm 41,6 $\mu \mathrm{m}$, el cual está en una rango cercano al de Anguilla japonica que es $350 \mathrm{~mm}$, en otras especies como Cyprinus carpio. En cuanto al número de fibras nerviosas, $C$. auratus presenta un número total de fibras de 120000 (Easter et al., 1981), número que supera al estimado en este estudio para el tiburoncito (22847 \pm 4350$)$. Sin embargo, los rangos de tamaños de fibras mielínicas encontrados en este trabajo (0.5$4,4.5-8$ y $<8 \mu \mathrm{m}$ ), son similares a los reportados para otras especies de vertebrados. En Perca fluviatilis se han reportado dos grupos de fibras $(0.2-3 \mu \mathrm{m}, 3-10 \mu \mathrm{m})$ (Guthrie \& Banks, 1990). En C. auratus se reportaron valores de 1.5, 3 y en $4 \mu \mathrm{m}$ (Easter et al., 1981). Eugerres plumieri presenta fibras con tamaño desde 0.2 a $7.6 \mu \mathrm{m}$ (Tapp, 1974). Danio rerio en promedio tiene fibras de $0.68 \mu \mathrm{m}$ de diámetro (Bakken \& Stevens 2011). En Pseudemys scripta elegans se reportan fibras de 2.8-4.5 $\mu \mathrm{m}$, y de $0.4-2.8 \mu \mathrm{m}$ (Woodbury \& Ulinski, 1986) y en Macaca fascicularis se ha reportado que las mayores densidades de fibras se encuentran en diámetros de 0.55, 0.85 y $1.55 \mathrm{~mm}$, (Sanchez et al., 1986).

En términos de velocidad de conducción, se ha señalado que, en general, la mayoría de las fibras del nervio óptico tienen en promedio velocidades de conducción bajas, entre 2.2 a $5.1 \mathrm{~m} / \mathrm{s} 2$ (Northmore \& Oh, 1998; Bakken \& Stevens 2011). Adicionalmente, la literatura plantea que el nervio óptico tiene pocas fibras con mayor velocidad de conducción y que, probablemente, están asociadas con fibras eferentes que conducen señales del cerebro hacia la retina, puesto que se ha descrito que en teleósteos estas fibras retinopetales tienen tamaños del orden de 1-3 $\mu \mathrm{m}$ (Northmore \& Oh 1998; Reperand et al., 2006). Diversos autores (apud Reperand et al., 2006) sugieren que estas fibras pueden pro- venir del sistema olfatorio y modular la sensibilidad al contraste de colores, o que también que corresponden a fibras que llevan impulsos táctiles.

En referencia a las otras fibras de baja velocidad de conducción, se reporta que las más rápidas de estas proyectan al tectum y hacen sinápsis superficiales, mientras que las más lentas lo hacen más profundamente (Schmidt, 1979). En general las fibras del nervio óptico inervan el tectum óptico (TO) desde la región media hasta la posterior (Johnson et al., 1999). Específicamente la región ventronasal de la retina inerva el tercio medio o el tercio posterior del tectum óptico y la lamina óptica en goldfish (Dawson \& Meyer, 2008). Esto sugiere que las diferentes subpoblaciones de fibras en el nervio óptico de $A$. seemanni podrían corresponder a la inervación en diferentes regiones del tectum, generando una topografía específica en esa estructura. Esto se relaciona con la estructura organizada en láminas en el tectum de peces (Nguyen et al., 1999), y la existencia de al menos cuatro subláminas que reciben fibras de las células ganglionares de la retina (Sanes \& Zapursky, 2010). Esta segregación podría estar también relacionada con una distribución diferencial de las células ganglionares de la retina. Se ha descrito en goldfish que la mayoría de células ganglionares presentes en el centro de la retina tienen un diámetro entre 7 y $8 \mu \mathrm{m}$, mientras que en la parte intermedia tienen un diámetro de $8-9 \mu \mathrm{m}$ y en la región periférica de la retina se encuentran neuronas entre 9 y $10 \mu \mathrm{m}$ de diámetro (Devadas et al., 2000), lo cual muestra una segregación de neuronas por tamaño a lo largo de la retina.

En conclusión el nervio óptico de A. seemanni tiene la conformación histológica típica de vertebrados. Presenta fibras mielínicas, en medio de las cuales se encuentran los septos de tejido conectivo delimitando fascículos de fibras nerviosas, y los septos gliales con oligodendrocitos y astrocitos. El nervio óptico está recubierto por las meninges (piamadre, aracnoides y la dura madre); compuestas por tejido conectivo en las cuales se localizan vasos sanguíneos y adipositos. Las fibras mielínicas presentan diferentes áreas y diámetros, encontrándose una mayor proporción de fibras pequeñas, que puede estar relacionado con velocidades de conducción bajas; en menor proporción se encontró fibras de tamaños grandes que podrían relacionarse con fibras eferentes.

\section{AGRADECIMIENTOS}

Agradecemos a la Vicerrectora de Investigaciones de la Universidad Militar Nueva Granada por el apoyo financiero proporcionado para la realización de este proyecto CIAS 163. 
CASTAÑEDA, C. D. C.; TOVAR, B. O \& HURTADO, G. H. Morphological study of tete sea catfish (Ariopsis seemanni) optic nerve. Int. J. Morphol., 31(1):184-188, 2013.

SUMMARY: The optic nerve carries out the information integrated by retinal ganglion cells towards the optic tectum, so that surrounding environment images are generated. The main goal of this paper is to describe morphometricaly the Tete sea catfish (Ariopsis seemanni) optic nerve, using high resolution optical microscopy (HROM), on 1 micron thick sections. A. seemanni optic nerve does present myelinated nerve fibers, accompanied by oligodendrocytes and astrocytes. The nerve is covered by the meninges, with blood vessels and adipocites. The nerve has a transversal area of 179,604 $\pm 30163 \mathrm{~mm} 2$, a diameter of $478 \pm 42 \mathrm{~mm}$ and $22848 \pm 4350$ myelinated fibers, most are small in size, which may be related to a low conduction velocity.

KEY WORDS: Tete sea catfish; Optic nerve; Nerve fibres; Morphometry.

\section{REFERENCIAS BIBLIOGRÁFICAS}

Akaishi, Y.; Uchiyama, H.; Ito, H. \& Shimizu, Y. A morphological study of the retinal ganglion cells of the Afghan pika (Ochotona rufescens). Neurosci. Res., 22:112,1995 .

Bakken, T. E \& Stevens C. F. Visual System Scaling in Teleost Fish. J. Comp. Neuro., 520:142-53, 2012.

Bolton, S. \& Butt, A. M. The optic nerve: A model for axonglial interactions. J. Pharmacol. Toxicol. Methods, 51: 22133, 2005.

Bussing, W. A. Peces de las Aguas Continentales de Costa Rica. Ed. Universidad de Costa Rica, San José, 1998.

Butler, A.B. \& Hodos, W. Comparative Vertebrate Neuroanatomy Evolution and Adaptation. 2 ed. WileyInterscience, New York, 2005.

Butt, A. M.; Colquhoun, K.; Tutton, M. \& Berry, M. ThreeDimensional morphology of astrocytes and oligodendrocytes In The Intact Mouse Optic Nerve. $J$. Neurocytol., 23:469-85, 1994.

Caldas, M. L.; Ricaurte, O.; Rodríguez, G. \& Amaya, J. Microscopía óptica de alta resolución "MOAR". Instituto Nacional de Salud, Bogotá, 1993.

CCAC. Canadian Council on Animal Care. Guidelines on: the care and use of fish in research, teaching and testing. Ottawa, Ontario, Canada, Canadian Council on Animal Care, 2005.

Collin, S. P. \& Shand, J. Retinal Sampling and the Visual Field in Fishes. Pp. 139-170. En: Collin, S.P. \& Marshall, J.N. (eds.). Sensory Processing in Aquatic Environments. Springer. Nueva York, 2003.

Dawson, A. J. \& Meyer, R. L. Growth dynamics and morphology of regenerating optic fibers in tectum are altered by injury conditions: An in vivo imaging study in goldfish. Exp. Neurol., 210: 592-601, 2008.
Devadas, M.; Sugawara, K.; Shimada, Y.; Sugitani, K.; WuLiu, Z.; Matsukawa, T. \& Kato, S. Slow recovery of goldfish retinal ganglion cells' soma size during regeneration. Neurosci. Res., 37:289-97, 2000.

Donovan, A. The nerve fibre composition of the cat optic nerve. J. Anat., 101:1-11, 1967.

Easter, S. S.; Rusoff, A. C. \& Kish, P. E. The growth and organization of the optic nerve and tract in juvenile and adult goldfish. J. Neurosci., 1(8):793-811, 1981.

Fernald, R. D. Sensory systems. En: Ostrander, G. The Laboratory Fish. Academic Press. Nueva York, 2000.Pp.225-233, 451-462.

Fujita, Y.; Imagawa, T. \& Uehara, M. Comparative study of the lamina cribrosa and the pial septa in the vertebrate optic nerve and their relationship to the myelinated axons. Tissue Cell, 32(4):293-301, 2000.

Guthrie, D. M. \& Banks, J. R. A correlative study of the physiology and morphology of the retinotectal pathway of the perch. Visual Sci., 4:367-77,1990.

Hitchcock, P. F. \& Easter, S. S. Retinal ganglion cell in goldfish: a qualitative classification into four morphological types, and a quantitative study of the development of one of them. J. Neurosci., 6(4):1037-050, 1986.

Hoppe, D.; Bastmeyer, M.; Von-Blankenfeld, G.; Kettenmann, H. \& Stuermer, C. A. O. Two populations of glial cells from fish optic nerve/tract with distinct electrophysiological properties. Exp. Brain Res., 87:383-8, 1991.

Johnson, F. A.; Dawson, A. J. \& Meyer, R. L. Activity-dependent refinement in the goldfish retinotectal system in mediated by the dynamic regulation of processes withdrawal: an in vivo imaging study. J. Comp. Neurol., 406(4):548-62, 1999.

Maggs, A. L. \& Scholes, J. Reticular Astrocytes in the Fish Optic Nerve: Macroglia with Epithelial Characteristics Form 
an Axially Repeated Lacework Pattern, To Which Nodes of Ranvier Are Apposed. J. Neurosci., 10(5):1600-1614, 1990.

Nguyen, V.; Deschet, K.; Henrich, T.; Godet, E.; Joly, J. \& Wittebrodt, J. Morphogenesis of he Optic Tectum in the Medaka (Oryzias latipes): A Morphological and Molecular Study, With Special Emphasis on Cell Proliferation. J. Comp. Neurol., 413:385-404, 1999.

Northmore, D. P. M. \& Oh, D. J. Axonal conduction velocities of functionally characterized retinal ganglion cells in goldfish. J. Physiol., 506 (1):207-17, 1998.

Repérant, J.; Ward, R.; Miceli, D.; Rio, J. P.; Médina, M.; Kenigfest, N. B. \& Vesselkin, N. P. The centrifugal visual system of vertebrates: A comparative analysis of its functional anatomical organization. Brain Res. Rev., 52(1):1-57, 2006.

Robertson, D. R. \& Allen, G. R. Shorefishes of the tropical eastern Pacific. Smithsonian Tropical Research Institute. Balboa, Panama, 2002.

Sanchez, R. M.; Dunkelberger, G. R. \& Quigley, H. A. The Number and Diameter Distribution of Axons in the Monkey Optic Nerve. Invest. Ophth. Vis. Sci., 27(9):1342-50, 1986.

Sanes, J. R. \& Zipursky, S. L. Design principles of insect and vertebrate visual systems. Neuron, 66:15-36, 2010.

Schmidt, J. T. The laminar organization of optic nerve fibers in the tectum of goldfish. Proc. R. Soc. Lond. B., 205:287306, 1979.
Tapp, R. L. Axon numbers and distribution, myelin thickness, and the reconstruction of the compound action potential in the optic nerve of the teleost: Eugerres plumieri. J. Comp. Neurol., 153:267-74, 1974.

Woodbury, P.B. \& Ulinski, P.S. Conduction velocity, size and distribution of optic nerve axons in the turtle, Pseudemys scripta elegans. Anat. Embryol., 174:253263, 1986.

Wullimann, M. F.; Rupp, B. \& Reichert, H. Neuroanatomy of de Zebrafish Brain a Topological Atlas. Birkhäuser Verlag. Berlin, Alemania, 1996.

Dirección para correspondencia:

Grupo de Ictiología

Programa de Biología Aplicada

Facultad de Ciencias

Universidad Militar Nueva Granada

Sede Campus Rio Grande

Cajicà Cundinamarca

COLOMBIA

Email: hernan.hurtado@unimilitar.edu.co

Recibido : 19-12-2011

Aceptado: 17-06-2012 